\title{
Management of Far-Advanced Otosclerosis: Stapes Surgery or Cochlear Implant
}

\author{
Original Investigation $>\begin{aligned} & \text { Münir Demir Bajin }{ }^{1} \text { (D), Onur Ergün² (D), Betül Çiçek Çınar }{ }^{3} \text { (D), Levent Sennaroğlu }{ }^{1} \text { (D) } \\ & { }^{1} \text { Department of Otorhinolaryngology-Head and Neck Surgery, Hacettepe University School of Medicine, Ankara, Turkey }\end{aligned}$ \\ ${ }^{2}$ Department of Otorhinolaryngology-Head and Neck Surgery, Ankara Training and Research Hospital, Ankara, Turkey \\ ${ }^{3}$ Department of Audiology and Speech Disorders, Hacettepe University School of Medicine, Ankara, Turkey
}

\section{Abstract}

\begin{abstract}
ORCID IDs of the authors: M.D.B. 0000-0003-1088-4367 O.E. $0000-0002-9252-0462$ B.C.C. 0000-0001-5496-3708 L.S. $0000-0001-8429-2431$.
\end{abstract}

Cite this article as: Bajin MD, Ergün 0 , Çınar BC, Sennaroğlu L. Management of Far-Advanced Otosclerosis: Stapes Surgery or Cochlear Implant. Turk Arch Otorhinolaryngol 2020; $58(1): 35-40$

\section{Corresponding Author:}

Onur Ergün, drergun@gmail.com

Received Date: 06.07.2019 Accepted Date: 30.11 .2019

Content of this journal is licensed under a Creative Commons Attribution 4.0 International License. Available online at www.turkarchotolaryngol.net
Objective: The aim of this report is to share our experience and treatment outcomes with far-advanced otosclerosis (FAO) patients.

Methods: Patients that underwent surgery from 2003 through 2014 at a tertiary referral center were retrospectively reviewed. Nineteen FAO patients were included in the study. Audiological results and the ability to communicate face to face and over telephone were considered as the main outcome measures.

Results: Six FAO patients benefited well from stapedotomy with an average of 5.9-decibel (dB) air-bone gap and $86 \%$ median speech discrimination. Cochlear implantation (CI) was performed in 13 patients; two had disease progression after stapedotomy, five had failed stapes surgeries elsewhere, and six preferred CI as primary treatment. Median speech discrimination score of CI patients was $78.4 \%$. Overall, all patients had satisfactory face-to-face communication and $90 \%$ could use telephone.

Conclusion: Bilateral stapedotomy and wearing hearing aid is an effective and cost-effective solution for restoring natural binaural hearing and requires no specific training. Should stapedotomy fail, cochlear implantation is always a successful back-up option.

Keywords: Cochlear implantation, otosclerosis, hearing loss, stapes surgery

\section{Introduction}

Otosclerosis (OS) is a localized genetic disorder of the endochondral bone, resulting in conductive, mixed, or sensorineural hearing loss (1). Far-advanced otosclerosis (FAO) was first defined by House and Sheehy (2) as an air conduction threshold of more than $85 \mathrm{~dB}$ with no measurable bone conduction threshold. The preferred imaging modality is high-resolution computerized tomography (HRCT). Medical treatment options could be used for early cases but surgical treatment options are necessary for most patients during follow-up. Various modifications of stapes surgeries yield excellent results in patients with primarily conductive hearing loss (1). For FAO, there are two major surgical options: stapes surgery with hearing aid, and cochlear implant (CI). Both approaches are proven to be effective and have different advantages or disadvantages over each other (3-5). In this study, we aimed to share our experience and treatment outcomes with FAO patients.

\section{Methods}

The authors assert that all procedures contributing to this work comply with the ethical standards of the national guidelines and with the Helsinki Declaration of 1975, as revised in 2008. Approval of the ethics board was obtained prior to the study (2014/354-15).

Patients with OS who underwent surgery from 2003 through 2014 were retrospectively reviewed. Patients who could not hear pure tones up to 110 decibel (dB), had no measurable bone conduction threshold, and a speech recognition (SR) level lower than $50 \%$ were included in the study. Patients were informed about the success rate, advantages and potential risks of the stapes and CI surgeries during the decision making process and informed consent including using their data for scholarly research were taken. They were informed that they would be able to use hearing aids and should the surgery fail, they would be con- 
sidered as CI candidates. Unless patients accept the possibility of a salvage surgery and prefer CI directly, according to the concept of our clinic, the correction of FAO is first attempted via stapedotomy, preferably bilaterally. Cochlear implantation is reserved for cases in which stapedotomy is unsuccessful or imaging techniques unequivocally reveal a fenestral or cochlear obstruction. Stapedotomy is not offered more than once if sufficient benefit could not be gained or if the patient had failed stapes surgery attempts. In this study, several parameters were taken into consideration, including age, gender, follow-up after surgery, and the ability to communicate over telephone. Postoperative sentence recognition and monosyllable word test scores were obtained in all patients.

\section{Statistical Analysis}

The IBM Statistical Package for Social Sciences for Windows version 20.0 (IBM SPSS Corp.; Armonk, NY, USA) software was used for statistical analysis and calculations. Due to the low number of cases, non-parametric Mann-Whitney U test was used in comparative calculations.

\section{Results}

Out of 623 OS cases, 19 had FAO (3\%). The median age was 56 years (ranging from 24 to 81 years). Ten patients were male, nine were female. Male to female ratio was 1.7:1.5 The median follow-up period was 56 months (ranging from 18 to 180 months).
Some patients had known OS for more than 50 years and had undergone multiple stapes surgeries.

Eight FAO patients (42.1\%) underwent stapedotomy as initial surgery. All patients were fitted with $0.6-\mathrm{mm}$ diameter fluoroplastic prosthesis. None of the patients had middle ear anomaly. Out of the eight FAO patients who underwent stapedotomy, two were also noted to have biscuit footplates. The only intraoperative complication in the stapedotomy group was perilymph oozing in one patient that led to total sensorineural hearing loss and eventually necessitated CI. Additionally, perilymph gusher was encountered in the same patient during CI. Two patients (25\%) did not benefit from stapedotomy and eventually had CI. Follow-up period of the patients who benefited from stapedotomy ranged from 22 to 145 months with the median of 40.5 months. All patients that benefitted from stapedotomy could communicate well, both face-to-face and over telephone. Retrospective analysis of the stapedotomy cases was challenging because pre- and post-operative audiological assessment of some patients' previous operations were incomplete. The postoperative results given in Table 1 belong to their last operated ear. Postoperative SR scores of the six patients who benefitted from stapedotomy ranged from $75.9 \%$ to $97.5 \%$, with a median of $86 \%$, and monosyllable word recognition (MWR) scores ranged from $20 \%$ to $92 \%$, with the median of $34 \%$. Air-bone gap (ABG) levels decreased from $37.45 \mathrm{~dB}$ to $5.8 \mathrm{~dB}$ in average.

Table 1. Summary of demographic data and results. Speech recognition (SR) test and monosyllable word recognition (MWR) scores of the stapedotomy patients with hearing aids

\begin{tabular}{|c|c|c|c|c|c|c|c|c|c|}
\hline $\mathrm{n}$ & Age & Sex & $\begin{array}{l}\text { Follow-up time } \\
\text { after stapedotomy } \\
\text { (months) }\end{array}$ & $\begin{array}{c}\text { Previous } \\
\text { Stapedotomy } \\
\text { history }\end{array}$ & $\begin{array}{l}\text { Follow-up time } \\
\text { after CI } \\
\text { (months) }\end{array}$ & $\begin{array}{c}\text { Communication } \\
\text { over } \\
\text { telephone }\end{array}$ & $\begin{array}{l}\text { Post-op } \\
\text { Air-Bone } \\
\text { Gap }\end{array}$ & $\begin{array}{c}\text { Post-op } \\
1 \text { year SR } \\
\text { Test Score }\end{array}$ & $\begin{array}{c}\text { Post-op } \\
1 \text { year MWR } \\
\text { Test Score }\end{array}$ \\
\hline 1 & 64 & $\mathrm{M}$ & $30(\mathrm{U})$ & $\checkmark(\mathrm{U})$ & $18(\mathrm{U})$ & $\checkmark$ & & $93.7 \%$ & $44 \%$ \\
\hline 2 & 36 & M & $24(\mathrm{U})$ & $\checkmark(\mathrm{U})$ & 18(R), 12(L) & $\checkmark$ & & $97.5 \%$ & $48 \%$ \\
\hline 3 & 61 & $\mathrm{~F}$ & $24(\mathrm{~B})$ & & & $\checkmark$ & 3.75 & $97.5 \%$ & $48 \%$ \\
\hline 4 & 44 & $\mathrm{~F}$ & $36(\mathrm{~B})$ & & & $\checkmark$ & 8.75 & $86 \%$ & $20 \%$ \\
\hline 5 & 29 & $\mathrm{~F}$ & $22(\mathrm{~B})$ & & & $\checkmark$ & 5 & $92.4 \%$ & $44 \%$ \\
\hline 6 & 44 & $\mathrm{M}$ & $56(\mathrm{~B})$ & & & $\checkmark$ & 11.25 & $86 \%$ & $24 \%$ \\
\hline 7 & 59 & M & $45(\mathrm{~B})$ & & & $\checkmark$ & 1.25 & $79.7 \%$ & $92 \%$ \\
\hline 8 & 76 & $\mathrm{~F}$ & $145(\mathrm{~B})$ & & & $\checkmark$ & 5 & $75.9 \%$ & $76 \%$ \\
\hline 9 & 56 & $\mathrm{M}$ & & $x$ & $12(\mathrm{U})$ & $\checkmark$ & & $67 \%$ & too early \\
\hline 10 & 58 & M & & $\checkmark(\mathrm{U})$ & $14(\mathrm{U})$ & $\checkmark$ & & $66.8 \%$ & $36 \%$ \\
\hline 11 & 22 & M & & $x$ & $26(\mathrm{U})$ & $\checkmark$ & & $92.4 \%$ & $68 \%$ \\
\hline 12 & 42 & $\mathrm{~F}$ & & $\checkmark(\mathrm{B})$ & $75(\mathrm{U})$ & $\mathbf{x}$ (illiterate) & & $70 \%$ & $0 \%$ \\
\hline 13 & 74 & $\mathrm{M}$ & & $x$ & $87(\mathrm{U})$ & $x$ & & $31.6 \%$ & $0 \%$ \\
\hline 14 & 22 & M & & $x$ & $109(\mathrm{U})$ & $\checkmark$ & & $92.4 \%$ & $36 \%$ \\
\hline 15 & 55 & $\mathrm{~F}$ & & $\checkmark(\mathrm{U})$ & $117(\mathrm{U})$ & $\checkmark$ & & $78.4 \%$ & $12 \%$ \\
\hline 16 & 79 & M & & $\checkmark(\mathrm{B})$ & $136(\mathrm{U})$ & $\checkmark$ & & $34.1 \%$ & $0 \%$ \\
\hline 17 & 42 & $\mathrm{M}$ & & $x$ & $173(\mathrm{U})$ & $\checkmark$ & & $91 \%$ & $48 \%$ \\
\hline 18 & 66 & $\mathrm{M}$ & & $\checkmark(\mathrm{U})$ & $180(\mathrm{U})$ & $\checkmark$ & & $40.5 \%$ & $\begin{array}{l}\text { Insufficient } \\
\text { rehabilitation }\end{array}$ \\
\hline 19 & 58 & $\mathrm{~F}$ & & $x$ & $178(\mathrm{U})$ & $\checkmark$ & & $88.6 \%$ & $28 \%$ \\
\hline
\end{tabular}


CI was performed in 13 patients (65\%). Seven had prior failed stapes surgeries and six chose $\mathrm{CI}$ as the initial procedure. Full electrode insertion was successful in all cases (Figure 1). Two perilymph gusher and one oozing incidents occurred and were repaired immediately. Follow-up period after initial tune-up ranged from 12 to 180 months with the median of 87 months. No serious postoperative complications or side effects were noted during follow-up. All 13 patients could carry out face-to-face communication and 11 patients (84.6\%) could communicate over telephone. SR test scores ranged from $31.6 \%$ to $97.5 \%$, with the median of $78.4 \%$. MWR scores of our patients who had proper rehabilitation over one year ranged from $0 \%$ to $68 \%$ with a median of $29 \%$.

When we compared the SR test scores of 13 patients who had CI and six patients who benefitted from stapedotomy and hearing aids, there was no statistically significant difference $(\mathrm{p}=0.368)$. Similarly, there was no difference when we compared the SR results of the six patients who preferred CI as the primary treatment and the eight patients who had stapedotomy as the primary treatment and back-up CI if necessary $(\mathrm{p}=0.414)$.

In the long-term follow up, in total, $80 \%$ of our patients had SR levels higher than $66 \%, 90 \%$ were able to communicate over telephone, and all patients were able to carry out face-to-face communication. All results and some additional information are summarized in Table 1.

\section{Discussion}

FAO patients have an air conduction threshold of more than $85 \mathrm{~dB}$ with no measurable bone conduction threshold. In interpreting the audiogram of FAO patients, it should be considered that "sensorineural" hearing loss does not occur solely because of degenerating hair cells, but partially because of the rigid fixation of the annular ligament itself. There has to be at least two mobile windows for the perilymph to stay inert and therefore the bone thresholds may be determined. Otherwise, it is impossible to evaluate the "cochlear potential" non-invasively. Stapedotomy unlocks that hidden potential and patients once thought to have profound sensorineural hearing loss may benefit significantly. To

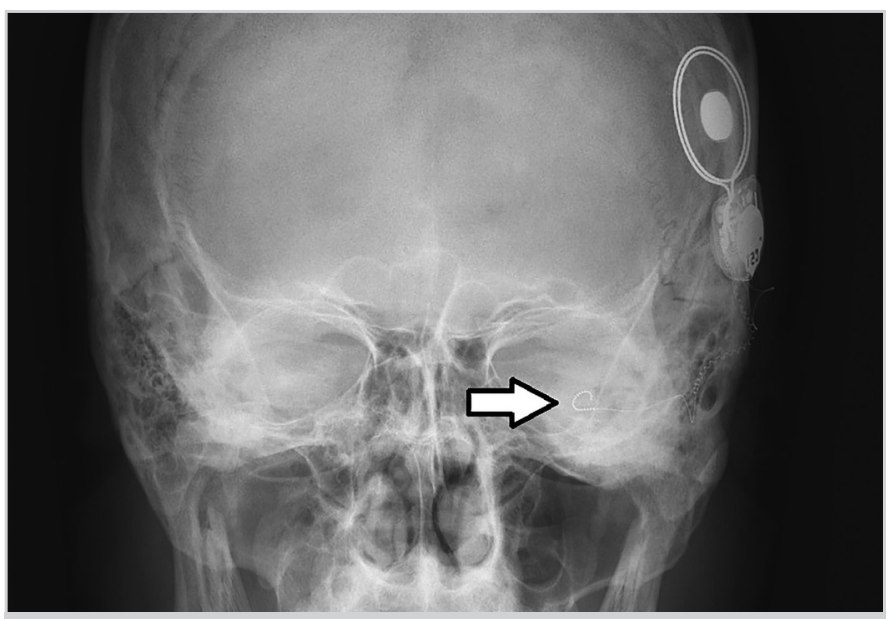

Figure 1. Full electrode insertion can be seen at the postoperative transorbital $\mathrm{x}$-ray with a modiolar hugging electrode better estimate cochlear reserve, electrocochleography (ECogG) could be used to measure the electrical responses of the inner ear and the auditory nerve (6).

There are four main treatment modalities for FAO. First, follow-up, medical therapy and hearing aids may be used especially in patients who are not suitable for surgery. Secondly, stapedotomy and hearing aid. Third, stapedotomy combined with active middle ear implants such as CodacsTM Direct Acoustic Cochlear Implant (DACI) system (Cochlear Ltd., Sydney, NSW, Australia) (4) or Vibrant Soundbridge (MEDEL Hearing Technology, Innsbruck, Austria) (5) and lastly, CI (6-8). However, there is not a widely accepted guideline for the decision-making process. Both stapedotomy and CI have different advantages and disadvantages, and thus the treatment modality should be tailored according to the patient's history, clinical signs and symptoms, audiological results (tuning fork tests, pure tone audiogram, speech threshold testing, SR), HRCT findings (Figure 2), ECoG/promontory stimulation and individual expectations.

Stapes surgery combined with active middle ear implants may offer a solution to moderate to severe OS cases who can not benefit hearing aids but have sufficient cochlear reserve. DACI was reported to provide superior speech reception in noise compared to CI (4). Vibrant sound bridge was also shown to be comparable to $\mathrm{CI}$ in moderate to severe OS cases (5). Unfortunately, invasiveness or limitations of eCoG may impede precise estimation of cochlear reserves in FAO cases (9). Additionally, the progressive nature of the condition may decrease the cochlear reserve to a point that a salvage $\mathrm{CI}$ is inevitable. The cost and complexity of the procedure are also important to mention.

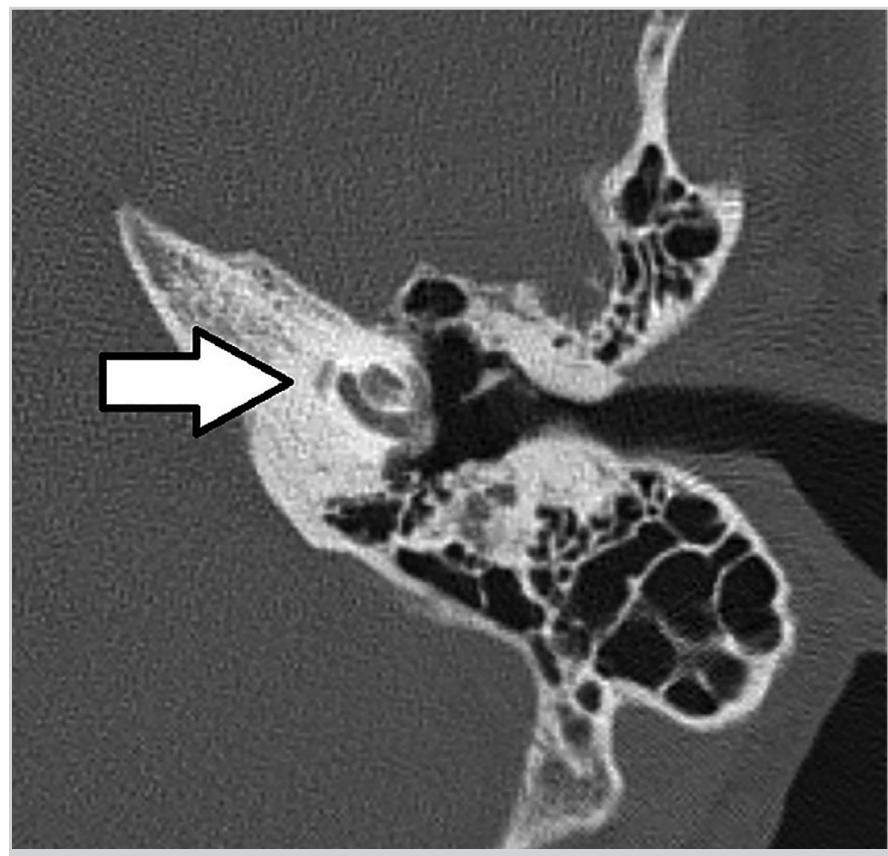

Figure 2. An axial section of high-resolution computed tomography shows diffuse peri-cochlear demineralization. This patient did not sufficiently benefit from the initial stapes surgery and cochlear implantation was done 
In the era of CI, SR scores are more frequently used than puretone thresholds for the follow-up of patients with severe-to-profound hearing loss $(6,10)$. Published SR scores of OS patients with CI range from $45 \%$ to $98 \%$. Many studies report better hearing results with $\mathrm{CI}$ than with stapes surgery (11-14). SR levels in our group ranged from $31.6 \%$ to $97.5 \%$, which is consistent with the literature. Moreover, all patients had good faceto-face communication and 11 patients (84.6\%) could communicate over telephone. We think that being able to use telephone, although not quantitative, is a good indicator of the functional hearing outcome and patient quality of life. That one of the two patients who could not communicate over the telephone despite an SR level of $70 \%$ and the ability to carry out normal face-toface communication was probably due to being illiterate. MWR test is perhaps the most difficult test for the patients to accomplish and usually the latest gained ability during rehabilitation (15). The median MWR score of our patients who had proper hearing rehabilitation at least for one year was 36\%. The MWR scores also seemed to be parallel to the success of face-to-face communication skills and communication over telephone.

On the other hand, $\mathrm{CI}$ is a more expensive procedure and requires a long-lasting rehabilitation period. Moreover, some authors reported disappointing results due to electrode insertion difficulties and facial nerve stimulation, especially in diffusely spongiotic advanced cochlear otosclerosis cases, most probably due to decreased impedances of the otic capsule and increased voltages to maintain the expected hearing gain $(5,10,12,13)$. Modiolar hugging electrodes may decrease the incidence of facial nerve stimulation in such cases. Dumas et al. (12) reported similar CI outcomes and surgical difficulty/complication rates with FAO cases using straight electrode arrays. No serious postoperative complications or side effects were noted during the follow-up of our cases. Nevertheless, informing patients about

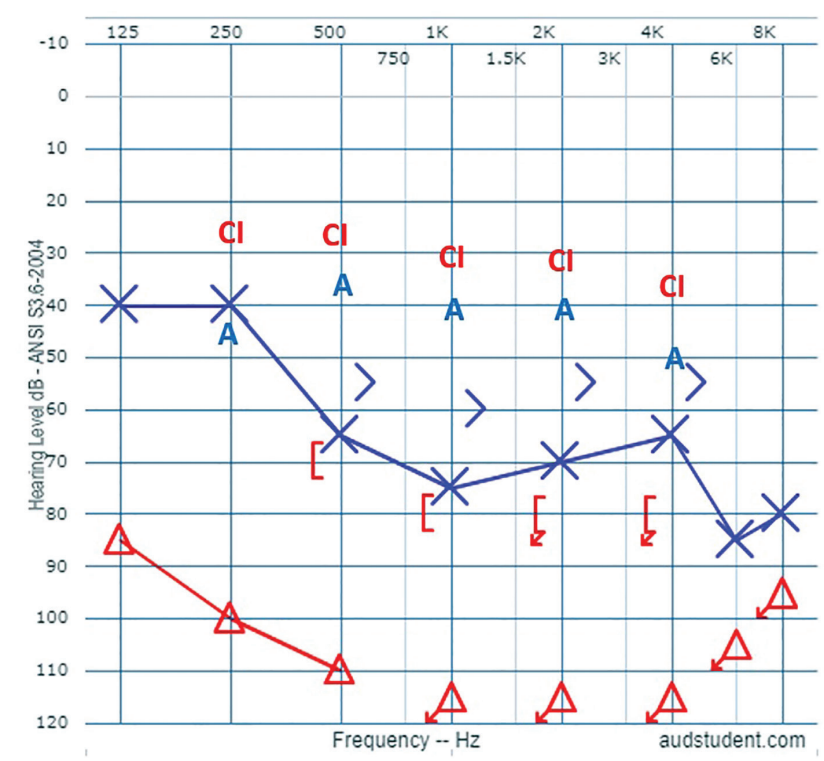

Figure 3. Pre- and post-operative audiograms of a right sided FAO that was treated with a cochlear implant. Left ear had also otosclerosis but sufficiently benefitted from hearing aid and did not undergo surgery. CI: postoperative thresholds, A: aided thresholds facial nerve stimulation and close follow-up is crucial. Figure 3 shows the pre- and post-operative pure tone audiogram results of a FAO case.

Alternatively, patients may be treated with stapedotomy and then fitted with hearing aids. Stapedotomy is a relatively simple, safe and low-cost procedure and can give acceptable outcomes in FAO $(14,16)$. Stapes surgery enables the acoustic stimulation of cochlea, providing a more natural sound perception than electrical stimulation of $\mathrm{CI}$ and does not require a long rehabilitation/fitting period which is crucial for CI users to benefit. SR scores of FAO after stapedotomy range from $38 \%$ to $75 \%$, which is slightly lower than CI results $(6,11,12)$. Nonetheless, stapedotomy can always be performed bilaterally and advantages of binaural hearing such as localization of sound or better speech discrimination in noise may be gained (10). In our group, six patients that benefitted from stapedotomy had even better hearing than the CI patients (Figure $4,5)$ with a median SR value of $86 \%$ and MWR score of $34 \%$. None of the patients had decreasing thresholds after successful stapedotomy nor needed CI in the follow-up period. Two patients did not benefit from stapedotomy at all and required CI. The performance after stapedotomy can sometimes be disappointing because of the difficulty in predicting the outcome of stapedotomy in patients with FAO $(11,14)$. All patients that benefitted from stapedotomy were later operated on their other ears and also had satisfying results. It is also worth mentioning that when the insufficient benefit and salvage CI surgery possibility of stapedotomy is mentioned, many patients who have suffered hearing difficulty for years tend to choose CI-the fastest route to hearing-even if that meant unilateral hearing. Six patients (31\%) made such a decision.

Lachance et al. (17) published a study in which they reported outstanding results of stapedotomy in a group of patients with

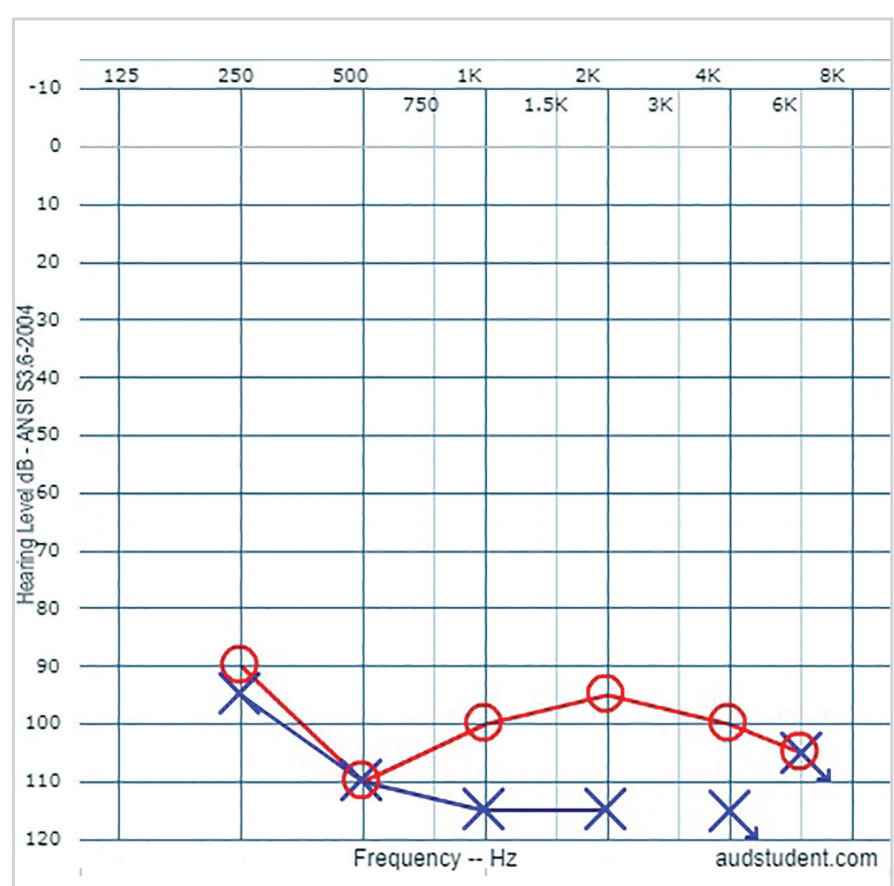

Figure 4. Preoperative audiogram of a FAO case 


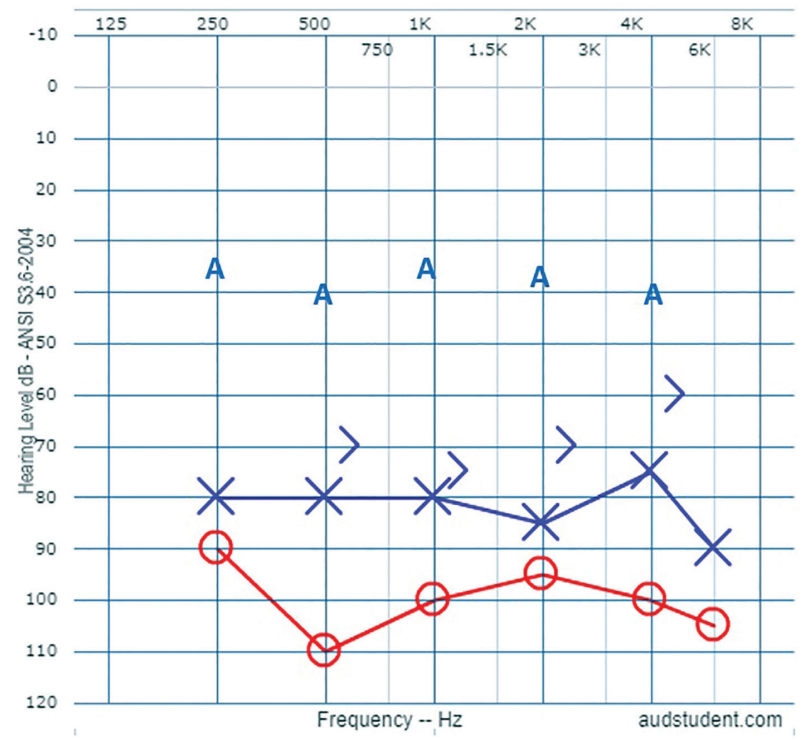

Figure 5. Postoperative audiogram of patient shown in Figure 4. Left side satisfactorily benefitted from stapedotomy and hearing aid. A: aided thresholds

FAO that were CI candidates. Eighty-seven percent of their patients had sufficient improvement in their air conduction thresholds and were no longer considered as CI candidates with the proper hearing aid use. Despite the reports of several publications in the last decade, there is no consensus on the outcome of stapedotomy in patients with FAO (10). In our series, stapedotomy and $\mathrm{CI}$ were equally effective considering the SR test results $(\mathrm{p}=0.368)$.

According to the concept of our clinic, the correction of FAO is first attempted via stapedotomy, although we acknowledge that most patients will eventually need CI. We do not consider a specific air-bone conduction threshold or discrimination score as a contraindication for stapedotomy. But contraindications of stapes surgery include some radiological findings such as fenestral/ cochlear opacification, round window obliteration or persistent stapedial artery.

At the time of this study, the social security system only covered unilateral CI for FAO patients. Therefore, bilateral CI could only be performed if the patient could afford the expenses related to the second CI surgery, and because most CI patients could not, instead of bilateral CI they had to settle for unilateral. Considering the financial limitations and the satisfying outcomes of stapes surgery, we perform bilateral stapedotomies whenever possible to maximize hearing gain and favor the advantages of binaural hearing. In this regard, we try to postpone the CI surgery as long as possible. Our approach may change in the future if bilateral CIs are covered under social security.

The SR test scores of the patients who went through our staged approach were similar to those who had CI as their primary therapy ( $\mathrm{p}=0.414)$. Seventy-five percent benefitted from stapedotomy and had the comfort of bilateral acoustic stimulation.
Eighty percent had SR levels above $66 \%$. Ninety percent were able to use telephone and all patients had sufficient face-to-face communication.

This study is retrospective in design and limited for the number of the cases. These limitations are mostly due to the rarity of the pathology, one that can be overcome by experience in time.

\section{Conclusion}

Both stapedotomy and CI are reliable primary treatment options for FAO with similar success rates. In our series, when stapedotomy was successful in one ear, the procedure resulted in $100 \%$ success on the other ear. Therefore, stapedotomy should be offered to every patient as the primary treatment modality of FAO and CI should be kept as a back-up solution. We believe that our phased approach guarantees a proper balance among treatment success, invasiveness and cost.

Informed Consent: Ethics committee approval was received for this study from the Ethics Committee of Hacettepe University (2014/354-15).

Informed Consent: Written informed consent was obtained from the patients who participated in this study.

Peer-review: Externally peer-reviewed.

Author Contributions: Concept - L.S.; Design - M.D.B., O.E., B.C.C.; Supervision - L.S.; Materials - M.D.B., O.E.; Data Collection and/or Processing - M.D.B., O.E., B.C.C.; Analysis and/or Interpretation - M.D.B., O.E.; Literature Search - M.D.B., O.E.; Writing M.D.B, O.E., B.C.C.; Critical Review - L.S.

Conflict of Interest: The authors have no conflicts of interest to declare.

Financial Disclosure: The authors declared that this study has received no financial support.

\section{References}

1. Ramsay HA, Linthicum FH. Mixed hearing loss in otosclerosis: indication for long-term follow-up. Am J Otol 1994; 15: 536-9.

2. House HP, Sheehy JL. Stapes surgery: selection of the patient. Ann Otol Rhinol Laryngol 1961; 70: 1062-8. [Crossref]

3. Berrettini S, Burdo S, Forli F, Ravecca F, Marcaccini M, Casani AP, et al. Far advanced otosclerosis: stapes surgery or cochlear implantation? J Otolaryngol 2004; 33: 165-71. [Crossref]

4. Kabbara B, Gauche C, Calmels MN, Lepage B, Escude B, Deguine $\mathrm{O}$, et al. Decisive criteria between stapedotomy and cochlear implantation in patients with far advanced otosclerosis. Otol Neurotol 2015; 36: e73-8. [Crossref]

5. Merkus P, van Loon MC, Smit CF, Smits C, de Cock AF, Hensen EF. Decision making in advanced otosclerosis: an evidence-based strategy. Laryngoscope 2011; 121: 1935-41. [Crossref]

6. Keith RW, Kereiakes TJ, Willging JP, Devine J. Evaluation of cochlear function in a patient with "far-advanced" otosclerosis. Am J Otol 1992; 13: 347-9.

7. Verhaert N, Borgers C, De Voecht K, Boon E, Desloovere C. A role for acoustic stimulation in advanced otosclerosis: Direct acoustic cochlear implant versus cochlear implant. Audiol Neurootol 2018; 23: 89-97. [Crossref] 
8. Chang CJ, Wen YH, Sun CH, Beltrame MA, Wu HP. Treatment of moderate-to-severe otosclerosis with simultaneous piston surgery and incus vibroplasty. Ci Ji Yi Xue Za Zhi 2019; 31: 96-101. [Crossref]

9. Marshall AH, Fanning N, Symons S, Shipp D, Chen JM, Nedzelski JM. Cochlear implantation in cochlear otosclerosis. Laryngoscope 2005; 115: 1728-33. [Crossref]

10. Munoz-Fernandez N, Morant-Ventura A, Achiques MT, Dualde-Beltran D, Garcia-Callejo FJ, Monrroy-Parada MV, et al. Evolution of otosclerosis to cochlear implantation. Acta Otorrinolaringol Esp 2012; 63: 265-71. [Crossref]

11. Roland PS, Roth L. Interinterpreter variability in determining the SP/AP ratio in clinical electrocochleography. Laryngoscope 1997; 107: 1357-61. [Crossref]

12. Van Loon M, Merkus P, Smit C, Smits C, Witte B, Hensen E. Stapedotomy in cochlear implant candidates with far advanced otosclerosis: A systematic review of the literature and meta-analysis. Otol Neurotol 2014; 35: 1707-14. [Crossref]
13. Calmels MN, Viana C, Wanna G, Marx M, James C, Deguine $\mathrm{O}$, et al. Very far-advanced otosclerosis: stapedotomy or cochlear implantation. Acta Otolaryngol 2007; 127: 574-8. [Crossref]

14. Sainz M, Garcia-Valdecasas J, Ballesteros JM. Complications and pitfalls of cochlear implantation in otosclerosis: a 6-year follow-up cohort study. Otol Neurotol 2009; 30: 1044-8. [Crossref]

15. Dumas AR, Schwalje AT, Franco-Vidal V, Bebear JP, Darrouzet $\mathrm{V}$, Bonnard D. Cochlear implantation in far-advanced otosclerosis: hearing results and complications. Acta Otorhinolaryngol Ital 2018; 38: 445-52.

16. Heining C, Banga R, Irving R, Coulson C, Monksfield P. Audiological outcome of stapes surgery for far advanced cochlear otosclerosis. J Laryngol Otol 2017; 131: 961-4. [Crossref]

17. Lachance S, Bussieres R, Cote M. Stapes surgery in profound hearing loss due to otosclerosis. Otol Neurotol 2012; 33: 7213. [Crossref] 